\title{
Nascita di un nome. Esercizi di memoria sul patrimonio immateriale della dieta mediterranea
}

The Birth of a name. Memory's exercises on the intangible heritage of the Mediterranean Diet

Elisabetta Moro

\section{OpenEdition}

\section{Journals}

Edizione digitale

URL: http://journals.openedition.org/aam/298

DOI: $10.4000 /$ aam.298

ISSN: 2038-3215

\section{Editore}

Dipartimento Culture e Società - Università di Palermo

Notizia bibliografica digitale

Elisabetta Moro, « Nascita di un nome. Esercizi di memoria sul patrimonio immateriale della dieta mediterranea », Archivio antropologico mediterraneo [Online], Anno XXI, n. 20 (1) | 2018, online dal 30 juin 2018, consultato il 28 décembre 2019. URL : http://journals.openedition.org/aam/298; DOI : 10.4000/aam.298

Questo documento è stato generato automaticamente il 28 dicembre 2019

Archivio antropologico mediterraneo 


\section{Nascita di un nome. Esercizi di memoria sul patrimonio immateriale della dieta mediterranea}

The Birth of a name. Memory's exercises on the intangible heritage of the Mediterranean Diet

Elisabetta Moro

\section{NOTE DELL'AUTORE}

Le interviste qui riportate sono in parte visibili sul sito www.granaidellamemoria.it alla voce Granai del Mediterraneo.

È l'intangibile a creare l'essenza delle cose e non il tangibile.

Lao Tse

La vera arte della memoria è l'arte dell'attenzione. Samuel Johnson

\section{Introduzione}

Questo articolo intende proporre alla comunità scientifica i primi materiali di una ricerca etnografica più ampia, tuttora in corso, che si pone quale obiettivo principale la raccolta e, in una fase successiva, l'eleborazione analitica delle testimonianze degli studiosi che per primi si sono occupati di Dieta Mediterranea partecipando al celebre studio epidemiologico comparativo che va sotto il nome di Seven Countries Study (1958-1983). Una ricerca cui la comunità scientifica biomedicale riconosce il merito di 
aver dimostrato, prima di altre, l'esistenza di una correlazione significativa tra le abitudini alimentari, gli stili di vita e i cosiddetti "fattori di rischio" che creano le precondizioni per lo sviluppo delle patologie cardio-cerebro-vascolari (Blackburn 2017). La ricerca in questione è stata scelta quale case study, in ragione del suo risvolto antropologico, in quanto gli autori non si limitarono ad analizzare parametri meramente biomedicali, come è stato ritenuto da molti, ma assunsero fin dall'inizio un punto di vista implicitamente antropologico.

2 L'intento generale da cui muove la presentazione di queste testimonianze, acquisite in formato audiovisuale, è quella di raccogliere e archiviare dei materiali etnografici il cui studio potrebbe supportare una sorta di urgent anthropology suggerita, se non altro, dall'età molto avanzata degli ultimi testimoni.

3 La ragione di questo lavoro di reperimento delle fonti vive, come le definisce Marc Bloch (1969), non nasce semplicemente dall'aspirazione di chi scrive di completare un lavoro sistematico sul patrimonio immateriale della Dieta Mediterranea iniziato nel 2010, vale a dire all'indomani del suo riconoscimento nella lista dei patrimoni immateriali dell'UNESCO, ma soprattutto dalla convinzione che sia possibile, e forse anche necessario, far emergere quanto l'approccio di questi ricercatori non fosse determinato da mere considerazioni medico-sanitarie (Teti 1999), ma avesse una spiccata coloritura antropologica, ancorché basata su una sensibilità politica e sociale, piuttosto che su un sostrato accademico scientifico, sostanziato da studi e approcci specifici.

Inoltre, nel corso del mio lavoro di ricerca, mi sono resa conto di come la genealogia del termine Dieta Mediterranea meritasse un'attenzione specifica, in quanto tale etichetta linguistica, oggi promossa allo statuto di topos - sia dal linguaggio comune, sia dal lessico della scienza, che da quello delle grandi agenzie internazionali come l'UNESCO, l'OMS e la FAO - di fatto comincia a diffondersi solo a partire dagli anni Settanta. Se in alcuni miei lavori precedenti, ho avanzato l'ipotesi che gli autori di questo nuovo conio lessicale fossero stati proprio Ancel e Margaret Keys, nel presente articolo intendo presentare per la prima volta le testimonianze che di fatto confermano tale ipotesi. Di qui la ragione del titolo di questo articolo Nascita di un nome. Esercizi di memoria sul patrimonio immateriale della Dieta Mediterranea. Si tratta, infatti, di far emergere in maniera quanto più chiara e densa possibile (Geertz 1987 [1973]) il contesto pubblico e privato in cui è maturata l'idea di attribuire un nome comune a delle forme di vita (Herder 1951 [1774], Remotti 1990, Moro 2014, Niola 2015) presenti nel bacino del Mediterraneo all'indomani della Seconda Guerra Mondiale.

5 In questo saggio intendo far emergere, attraverso una ricostruzione storicoepistemologica dei diversi passaggi che hanno contribuito a costruire tale nozione, la sua natura processuale e non sostanziale, nonché i dubbi, le perplessità e le controversie che hanno scandito le varie fasi delle attività del gruppo di ricerca.

6 La selezione, la natura e il numero delle testimonianze proposte in questa sede è stata dettata, oltre che dal criterio della pertinenza funzionale, anche dalla volontà di restituire non solo le informazioni necessarie a sostenere le tesi enunciate all'inizio di questo scritto, ma anche dall'intento di restituire, almeno in parte, ai diversi interlocutori la loro profondità individuale. I quali, va sottolineato, hanno acconsentito a dialogare con l'autrice, solo al termine di un lungo lavoro di accreditamento scientifico e personale presso quella che potremmo definire, per ragioni di sintesi, la "comunità del Seven Countries Study". Una comunità che si autorappresenta come un 
gruppo di "sopravvissuti" all'inesorabile trascorrere del tempo, ma anche agli attacchi mediatici, che da molti anni mettono radicalmente in questione la validità di questo studio. Determinando la nascita di un clima teso e a tratti diffamatorio, che ha spinto alcuni tra i maggiori scienziati della nutrizione mondiale, come Walter Willet dell'Università di Harvard e David L. Katz dell'Università di Yale, a pubblicare un Libro Bianco dal titolo Ancel Keys and the Seven Countries Study (2017) (pubblicato in Inglese sul sito www.treuhealthinitiative.org e www.unisob.na.it/medeatresearch e a breve in traduzione italiana sul sito www.elisabettamoro.com). Pertanto questi materiali vanno letti anche alla luce di un contesto di ricerca profondamente segnato da conflitti di ordine teorico, accademico, economico, nonché politico. Di conseguenza, in questa fase di sgrossatura del lavoro, l'approccio di chi scrive ha privilegiato l'ascolto degli informatori, rispetto alla decostruzione critica delle loro testimonianze, che potrà avvenire solo nelle fasi successive dell'intero progetto, quando i carteggi intercorsi fra chi scrive e la comunità del SCS, assieme alle memorie che alcuni testimoni hanno affidato all'autrice, saranno oggetto di ulteriore elaborazione.

7 Per molti altri temi e questioni rinvio ai miei lavori di ricerca precedenti $(2013,2014$, 2016, con Niola 2015).

\section{Il Seven Countries Study e la comparazione tra le culture}

8 I risultati del Seven Countries Study rimangono ancora oggi una pietra miliare della ricerca epidemiologica (Pett et al. 2017a, 2017b) e hanno ispirato buona parte delle politiche di salute pubblica dell'Occidente, in primis quelle dell'OMS, tanto che lo storico della medicina Arthur Stewart Truswell nel suo libro Cholesterol and beyond: The Research on the Diet and Coronary Heart Disease 1900-2000 ha definito lo studio delle Sette Nazioni «uno dei capitoli più avvincenti della storia della nutrizione del secondo dopoguerra» (2010: 183).

9 La tesi iniziale dello studio, ideato dal fisiologo dell'Università del Minnesota Ancel Keys, con l'aiuto della moglie, biologa della Mayo Foundation Margaret Haney e del fisiologo dell'Università di Napoli Gino Bergami, intese esplorare la possibile correlazione tra un elevato tasso di colesterolo nel sangue e l'insorgenza delle malattie cardio-vascolari (Moro 2014; Dixon 2015). Il campione esaminato per oltre trentacinque anni consecutivi, fu costituito di dodicimila maschi adulti sani, residenti in sette nazioni diverse: Italia, Grecia, Jugoslavia, Giappone, USA, Olanda e Finlandia. L'équipe internazionale costruita negli anni da Ancel Keys, di fatto inventò un metodo d'indagine pionieristico, a metà tra la medicina e una sorta di antropologia, mettendo insieme l'analisi dei corpi delle persone con osservazioni riguardanti una serie di dati relativi alla cultura di appartenenza. Una sintesi originale tra etnografia, seppur amatoriale, e anamnesi medica. Osservazione partecipante e analisi biochimica, supportate da una costante analisi statistica dei dati numerici e una altrettanto costante applicazione del metodo comparativo nella lettura dei dati più squisitamente etnografici. Dopo pochi anni dall'avvio dell'indagine, avviata nel 1958, iniziarono ad emergere chiaramente dei risultati che confermarono l'ipotesi di partenza: vale a dire che il modo di vivere e di alimentarsi tradizionali di tutti i paesi mediterranei (Italia, Grecia, Ex Jugoslavia) e anche del Giappone, manteneva bassi i livelli di colesterolo nel sangue e complessivamente favoriva condizioni di salute migliori. Dall'altra parte, 
invece, i soggetti analizzati residenti nei paesi con costumi nutrizionali basati su consumi sostenuti di grassi saturi e carni animali, risultarono avere una colesterolemia elevata e contemporaneamente si rivelarono maggiormente esposti alle malattie cardiovascolari (Keys et al. (1986) 2017; Kromhout et al. 1995; Blackburn 2017). Studi recenti hanno dimostrato inoltre che la dieta mediterranea non riduce solo l'insorgenza delle malattie cardio-vascolari, ma anche l'incidenza di quelle degenerative, come per esempio l'Alzheimer e molte altre (Solfrizzi et al. 1999; Boccardi et al. 2018), aumentando di fatto l'interesse scientifico internazionale per questo stile di vita e conseguentemente per lo studio che per primo ne ha decretato il valore positivo.

\section{Idealizzazione e narrazione di un modello culturale}

10 L'approccio del Seven Countries Study coniugò, di fatto, le più avanzate e oggettive rilevazioni di natura biomedicale, quali analisi del sangue, elettrocardiogrammi e monitoraggio della pressione arteriosa, con una rilevazione delle attività svolte durante la giornata, dei pasti consumati, delle tradizioni gastronomiche locali, dei sistemi di credenze, della produzione e dell'approvvigionamento del cibo. Insomma di tutti quegli elementi culturali che concorrono a costituire un sistema di pratiche, di poetiche, di retoriche riguardanti il rapporto tra alimentazione e benessere psicofisico. L'équipe internazionale arruolata da Ancel Keys, infatti, fu fin dall'inizio consapevole che la nutrizione svolge un ruolo rilevante, ma non è mai l'unico fattore in campo. Come mi hanno confermato due dei ricercatori del gruppo, Alessandro Menotti e Daan Kromhout, la vita sociale, l'habitat, la qualità degli alimenti, la varietà nutrizionale, $\mathrm{i}$ metodi di cottura, l'attività fisica, la salubrità dell'aria, assieme all'investimento simbolico che viene fatto sul cibo e molti altri elementi socio-culturali, anche se difficilmente misurabili, sono fattori che l'équipe di ricerca ha sempre considerato altrettanto determinanti per la qualità della salute delle persone (Kromhout 1995; Toshima 1994).

11 Se il sostrato antropologico venne per lo più sottaciuto nei testi più squisitamente scientifici, divenne invece preponderante nei tre libri divulgativi con cui Ancel e Margaret Keys fecero conoscere i risultati del SCS ad un pubblico più vasto. In Eat well and stay well (1959 edizione USA e Canada; 1960 edizione rivista per il Regno Unito), The benevolent bean (1967), How to eat well and stay well: The Mediterranean way (1975; edizione italiana 2017), infatti, i due scienziati non solo spiegarono dati alla mano quanto il mangiar sano incida sulla vita delle persone, ma sottolinearono l'importanza della convivialità, di una quotidianità fatta di relazioni sociali intense, dell'attività fisica all'aria aperta, del consumo regolare di frutta e verdura fresche e di stagione. Ed invitarono i loro lettori, per lo più appartenenti al mondo anglo-americano, a evitare il pane a lunga conservazione in quanto ricco di grassi idrogenati, per seguire l'esempio degli Italiani che, all'epoca, compravano il pane fresco quotidianamente. Raccontarono che con le verdure i popoli del Mediterraneo facevano capolavori gastronomici come la parmigiana di melanzane o la moussaka. Elogiarono gli agrumi della Sicilia e della Costiera Amalfitana, saporiti e ricchi di vitamine. Rievocarono lo stupore che li aveva colti la prima volta che avevano assaggiato una tortilla a Madrid, un fico d'india a Creta, un fico bianco in Cilento, la mozzarella di bufala a Caserta e il parmigiano a Bologna, trasformando l'aneddotica familiare in esempio generale. In curiosità etnografiche in grado di coinvolgere ed appassionare il lettori. Come quando raccontarono di portare 
con sé a Minneapolis l'olio extra vergine d'oliva biologico che producevano gli olivi del loro buen retiro cilentano di Pioppi. O quando elogiarono la pasta di Gragnano per le sue ottime qualità nutrizionali e ne consigliarono il consumo regolare, ricorrendo a retoriche particolarmente connotative, quasi da Scuola Medica Salernitana. Con una sapiente opera di idealizzazione e di costruzione di una narrazione improntata alla creazione di una nuova mitologia del Mediterraneo (Moro 2014: 39, 89, 211), i Keys trasformarono le culture locali in modelli ideali, da proporre ad un mondo avviato a grandi passi verso il consumo di junk food e verso uno modello sviluppo insostenibile. Così, pagina dopo pagina, i coniugi raccontarono la bellezza struggente delle dolci notti mediterranee e il fascino di quel paesaggio mitico che un tempo, amavano ripetere, fu attraversato da Ulisse. Insomma, parallelamente alle loro azioni più squisitamente pertinenti ai protocolli scientifici di ricerca, i Keys si fecero, sin dagli anni Sessanta, promotori di un modello culturale prima ancora che alimentare. Un Eden post atomico, dove l'Occidente potesse ritrovare se stesso (Moro 2014, 2016; Moro, Niola 2017).

\section{Divulgazione globale di un patrimonio immateriale locale}

12 Nell'ottica di Ancel e Margaret Keys, ma anche dei loro colleghi del SCS, la priorità della loro azione era operare nell'ambito della salute pubblica. Perciò, avendo dimostrato, almeno in via preliminare, che la dieta mediterranea aiuta a mantenersi in salute, la disseminazione dei risultati dello studio diventò presto una missione prioritaria. I risultati mediatici non si fecero attendere. Due esempi per tutti. Il Time pubblicò una lunghissima intervista ad Ancel Keys, alle sue ipotesi e agli esiti delle sue ricerche, e lo consacrò star della ricerca internazionale dedicandogli la sua celebre copertina listata di rosso nel gennaio del 1961 (Moro 2014: 169-179).

Nel 1985 la BBC dedicò alla coppia due puntate di un programma educational condotto dal medico inglese Michael O'Donnell intitolato The food connection. I Keys vennero ripresi durante un pranzo tipicamente mediterraneo nel naos del tempio di Hera a Paestum. A tavola sedeva anche Martii Karvonen, medico dell'esercito finlandese in pensione, incaricato dal governo del suo paese di progettare un piano di salute pubblica ispirato alle scoperte del Seven Countries Study (Moro 2014: 99-101). Inoltre era presente Anna Ferro-Luzzi, allora direttrice dell'Istituto Superiore di Sanità e autrice di una ricerca fondamentale dal titolo "Cilento Cuore", dedicata all'analisi degli effetti sulla colesterolemia della dieta cilentana comparata a quella dei Finlandesi, all'epoca considerata la più grassa al mondo (Ferro-Luzzi et al. 1984). Il servizio della BBC raccontò al grande pubblico le modalità di svolgimento di questa ricerca, entrando nelle case delle coppie esaminate, mostrando, insieme alle procedure scientifiche seguite dall'équipe, gli usi e i costumi locali. Ad Anna Ferro-Luzzi va inoltre attribuito il primato di avere usato per la prima volta l'espressione dieta mediterranea nella letteratura scientifica, in un saggio intitolato Changing the Mediterranean diet: Effects on blood lipids, apparso sull'American Journal of Clinical Nutrition nel 1984, dove illustrò proprio i risultati dell'esperimento cilentano. Il concetto di dieta mediterranea all'epoca era talmente poco diffuso nella comunità scientifica, che nella premessa del saggio Ferro-Luzzi e gli altri autori - come mi hanno personalmente confermato alcuni di loro: Pasquale Strazzullo e Alfonso Siani - avvertirono la necessità di delineare l'ambito geografico e i tratti caratteristici di tale stile nutrizionale, sottolineando, tra 
l'altro, che si trattava di un insieme di pratiche alimentari presenti in molti luoghi del Mediterraneo, che privilegiano il consumo di cereali, frutta e verdura.

Quando recentemente ho intervistato Anna Ferro-Luzzi per il progetto di ricerca 'Granai della Memoria', di cui dirigo la sezione 'Granai del Mediterraneo', la studiosa mi ha spiegato che l'espressione dieta mediterranea era di fatto nuova e forse anche un po' restrittiva, a causa del ricorso alla nozione di dieta che può trarre in inganno e far pensare solo ad un mero regime alimentare ipocalorico e non, invece, ad un vero e proprio stile di vita come va correttamente intesa. All'epoca però, sia Ferro-Luzzi che Ancel Keys, mentore e ispiratore anche se non autore della ricerca, giudicarono indispensabile utilizzare un'etichetta spendibile sul piano internazionale e facilmente comunicabile. Perciò optarono per l'utilizzo di quell'espressione che Ancel e Margaret Keys avevano coniato una decina di anni prima e lanciato nel 1975 nel loro best seller How to Eat Well and Stay Well. The Mediterranean Way (trad. It. 2017).

In conclusione, l'intervista del Time ad Ancel Keys e il documentario della BBC dedicato al simposio mediterraneo di Paestum costituiscono due momenti fondamentali nella storia della fortuna nella comunicazione pubblica sia del nome che del concetto di dieta mediterranea. Nel contempo la ricerca scientifica svolta in Cilento da Anna Ferro-Luzzi e dai suoi colleghi, assieme all'articolo che ne seguì, rappresentano un momento fondamentale nella storia delle scienze della nutrizione e, quel che più importa in questa sede, un campo antropologico di grande interesse sociale e politico. Questi tre momenti vanno intesi come dei tornanti nella genealogia della dieta mediterranea e occupano un posto di primo piano nell'intera vicenda della sua individuazione scientifica e della sua decodifica culturale. Inoltre, a mio avviso, essi costituiscono tre pietre miliari che segnano significativamente il continuum memoriale di questo patrimonio dell'umanità.

\section{Protagonisti e testimoni della "scoperta" della dieta mediterranea}

16 La dieta mediterranea, come è noto, è stata inscritta nel 2010 dall'UNESCO nella lista del Patrimonio Culturale Immateriale dell'Umanità, vale a dire in un elenco di "capolavori della cultura" che, secondo la Convenzione unescana del 2003, non sono fatti solo di cose, ma soprattutto di idee, storie collettive, tradizioni, pratiche sociali, sistemi di valori condivisi, mentalità e soprattutto di memorie (Petrillo 2018). Proprio per salvaguardare questo patrimonio di memorie il MedEatResearch, il Centro di ricerche sociali sulla dieta mediterranea dell'Università di Napoli SOB, che dirigo con Marino Niola, a partire dal 2010 ha iniziato una raccolta di testimonianze dei pionieri della dieta mediterranea, cioè di quelle donne e quegli uomini che hanno contribuito a farla, a scoprirla, a tramandarla, a farla conoscere al mondo. I protagonisti di queste interviste sono scienziati e persone comuni, cuoche e pescatori, intellettuali e contadini, perché un patrimonio come questo è necessariamente una costruzione collettiva e in continuo divenire. Tutte le testimonianze vengono regolarmente pubblicate sul sito www.unisob.na.it/medeatreserch e sul portale www.granaidellamemoria.it nella sezione "Granai del Mediterraneo di UNISOB" che è parte di un ampio progetto, diretto da Piercarlo Grimaldi, sulle memorie alimentari che l'Ateneo napoletano sta conducendo in collaborazione con l'Università di Scienze Gastronomiche di Pollenzo (Bra) e con Slow Food International. I Granai della Memoria 
hanno vinto il Premio dell'Unione Europea per il Patrimonio Culturale / Europa Nostra Awards 2016, la più alta onorificenza presente in Europa nel settore del patrimonio culturale e naturale. Nella motivazione si legge: «l'ampia e completa selezione del progetto ha il potenziale per farlo considerare su una più ampia scala e deve essere preso in considerazione in Europa come un esempio di buona pratica nella conservazione dei patrimoni immateriali. Questo progetto è un'occasione per offrire un Premio alla memoria delle persone comuni».

Estratti di queste interviste vengono pubblicati regolarmente sul canale YouTube del MedEatResearch con l'intento di rafforzare nell'opinione pubblica l'idea che molta parte del patrimonio culturale legato alla tavola è di natura orale e pertanto necessita di un lavoro di archiviazione puntuale (Grimaldi, Porporato 2012; Grimaldi 2012), nonché di una disseminazione capillare, che peraltro va intesa anche nel quadro della "terza missione" dell'università promossa dal MIUR.

Propongo dunque qui di seguito i miei colloqui con Carrie Keys, figlia di Ancel e Margaret, Henry Blackburn, cardiologo del SCS e Alessandro Menotti medico e statistico del SCS, pubblicate integralmente sul sito dei Granai della Memoria. Si tratta di tre testimonianze di primaria rilevanza storico-antropologica per comprendere $\mathrm{i}$ risvolti etnografici, sociali e umani legati alla individuazione e alla narrazione della dieta mediterranea.

\section{La figlia di Ancel e Margaret Keys}

A Minneapolis nel marzo 2015 ho intervistato Caroline Keys D'Andrea, con la collaborazione dell'antropologa Rossella Galletti, all'epoca dottoranda di ricerca in "Identità e pratiche di distinzione sociale attraverso il consumo alimentare". Il racconto della primogenita dei coniugi Keys, classe 1940, psicologa clinica, risulta prezioso perché in presa diretta con la storia. Se nelle forme di vita, infatti, rimangono impresse le impronte di quelle fonti vive che sono il cuore pulsante del patrimonio immateriale (Bloch 1969), nella biografia di Carrie le parole dei Keys hanno impresso segni indelebili, che in un primo momento però, complice il pudore, sembravano affiorare a fatica. Le sue indecisioni, titubanze e persino il rossore che affiorava sulle sue gote, mi lasciavano intendere che fosse la prima volta che rispondeva a delle domande sulla vicenda scientifica dei suoi genitori. Ad ogni risposta mi è parso di assistere all'affioramento di ricordanze fino ad allora taciute.

I tuoi genitori sono stati definiti i Marco Polo della Nutrizione per la loro ricognizione degli usi e costumi alimentari nel mondo, in particolare nel Mediterraneo. Hanno coinvolto tutta la famiglia?

Quando i miei genitori hanno scritto il loro best seller Eat well and stay well (1959), mi hanno arruolata come editor e come assaggiatrice. Allora ero una teenager e mia madre mi chiedeva di testare le ricette del loro libro, con il quale volevano spiegare ai nostri connazionali che cosa fosse la cultura alimentare del Mediterraneo, in particolare dell'Italia, della Grecia, della Spagna e del Midi della Francia.

In che modo le scoperte dei tuoi genitori hanno influenzato la tua cultura alimentare?

Nella mia famiglia si è sempre prestata molta attenzione al cibo. Le ricerche e gli insegnamenti dei miei genitori sui benefici di un'alimentazione con pochi grassi e poco sale, poca carne, ma con abbondanza di frutta e verdura fresca, sono state il 
nostro pane quotidiano. In famiglia, sia io che mio marito e i nostri figli, continuiamo a cucinare in casa, si dice "cooking from scratch" e si differenzia da quello che fa la maggior parte degli Americani che si sono convertiti ai cibi pronti, in scatola, surgelati e precotti.

Una delle regole di tuo padre consisteva in una grande varietà alimentare. II suo suggerimento era: Mai mangiare la stessa pietanza più di una volta nell'arco di due settimane. Seguivate davvero questa regola?

Mia madre era una cuoca eccellente. Cucinava moltissimi piatti diversi e questa regola delle due settimane era d'obbligo. Uno dei piatti più frequenti era pasta $\mathrm{e}$ fagioli. Lo aveva imparato in Italia. Ma faceva anche i fagioli stufati alla bostoniana (Boston baked beans), venivano cotti per molte ore con melassa, mostarda, un pizzico di sale e un po' di maiale. È un pasto tradizionale della costa orientale degli Stati Uniti. Comunque è vero che la nostra dieta quotidiana era molto varia: cibo italiano, cinese, svedese, americano. I nostri pasti migliori erano quelli delle feste. In quelle occasioni aveva la meglio il ritualismo di papà. Mangiavamo rigorosamente i piatti della tradizione americana come l'agnello arrosto a Pasqua, il tacchino il giorno del Ringraziamento e a Natale. Per Capodanno era fisso il salmone del Pacifico al forno.

La vostra educazione alimentare era solo nutrizionale?

Seduti a tavola parlavamo sempre di molte cose che giravano intorno al piatto, non solo dei nutrienti, e spesso venivamo invitati a prendere l'enciclopedia e a leggere delle voci tra una portata e l'altra.

Qual era il piatto preferito di tuo padre?

La pasta.

Di tua madre?

La lasagna, le alici fritte e un contorno a base di patate e carciofi.

Pensi che siano stati i tuoi genitori a inventare l'espressione dieta mediterranea?

Così mi è sempre stato detto. Ricordo che quando nel 1975 scrissero il terzo libro How to Eat Well and Stay Well: The Mediterranean Way furono invitati a usare questa espressione e ad inserire nel testo le ricette italiane che avevano imparato vivendo in Cilento proprio dall'editore Doubleday di Garden City N.Y.,

Che cosa significava per loro la dieta mediterranea?

Loro intendevano riferirsi allo stile di vita delle popolazioni che vivono lungo le coste del mar Mediterraneo, soprattutto di Italia, Grecia, Jugoslavia, Andalusia e della Francia meridionale. Ma anche dei paesi mediorientali che si affacciano sul mare. In questi luoghi loro hanno catalogato gli usi e costumi locali, nonché raccolto moltissime ricette dalla gente comune. Di queste una buona parte è stata inserita nei loro libri. Di fatto ammiravano quella storia e quella cultura alimentare, per i suoi sapori, per la sua salubrità, ma anche perché era un'alimentazione sana e sostenibile.

E per te?

Era la ragione di continue prese in giro da parte dei miei compagni di scuola. Perché quando mio padre cominciò a promuovere la dieta mediterranea negli States le industrie lattiero casearie e i produttori di carne non ne erano affatto contenti e sollevarono molti dubbi e polemiche. Così, nel senso comune, lui diventò il nemico dei grassi animali. E quando nella mensa i miei amici mi chiedevano di passargli il burro, mi dicevano "per favore, passami il veleno!". 
I tuoi genitori non sono vissuti abbastanza a lungo per assistere al riconoscimento UNESCO della Dieta Mediterranea, che cosa credi ne avrebbero pensato?

Sono certa che ne sarebbero stati felici. Sono anche sicura però che mio padre avrebbe detto che c'è ancora molta ricerca da fare in questo ambito. E molto lavoro per promuovere e disseminare i risultati delle ricerche scientifiche sull'alimentazione. Per migliorare lo stile di vita di molti paesi e non solo degli Stati Uniti, perché ci sono molte altre nazioni dove ci si alimenta molto male. La fissazione dei miei genitori era quella di rendere il mondo un posto migliore. Anche per questo i loro studi sono stati pionieristici, perché negli anni Cinquanta nessuno ragionava in termini di popolazione, erano gli albori dell'epidemiologia. E la loro idea di scoprire un modo per prevenire le malattie, anziché curarle, è stata una piccola rivoluzione.

Per il loro buen retiro avrebbero potuto scegliere qualunque posto del Mediterraneo. Invece hanno individuato proprio il villaggio di pescatori di Pioppi, nel comune di Pollica (Salerno) che è il cuore del Cilento. Oggi Pollica, grazie a questa loro scelta, è stata riconosciuta quale comunità emblematica dall'UNESCO. Perché proprio lì?

Lavorando molto a lungo in Italia si erano innamorati del Paese e soprattutto del Mezzogiorno. Amavano quella zona per il panorama, per il rapporto strettissimo tra mare e montagna, per la pace e la tranquillità. La gente poi era incredibilmente amichevole e ospitale.

Quando sono andata per la prima volta a fargli visita nella loro nuova casa, doveva essere il 1964, rimasi colpita dalla povertà di Pioppi e dal fatto che un solo edificio nel paese aveva l'elettricità. Gli abitanti conducevano una vita rurale molto arcaica e autentica. E poi le persone erano davvero speciali.

Per loro era più importante mangiare in maniera sana o mangiare in compagnia?

Le due cose erano inscindibili. Quando c'era una festa, non si tiravano mai indietro. A Pioppi per il giorno del Ringraziamento invitavano la gente del posto per il tradizionale tacchino. Mio padre trinciava e distribuiva le parti, mia madre sfornava il pane fatto in casa. Per loro la convivialità era molto importante.

Tuo padre è morto poco prima di compiere 101 anni e tua madre all'età di 97 anni. La dieta mediterranea è stata il loro elisir di lunga vita?

Il lavoro di ricerca è stato per loro uno stimolo enorme. Mio padre di fatto ha smesso solo due anni prima di andarsene. Erano entrambi molto attivi e hanno avuto cure mediche di ottimo livello, sia in Italia che negli USA. Il resto lo ha fatto l'alimentazione.

\section{Il successore di Ancel Keys}

Henry Blackburn, professore emerito dell'Università del Minnesota, ha collaborato a tutte le ricerche di Ancel Keys sulla dieta mediterranea a partire dal 1956. Nel 1972 ne ha preso il posto alla guida del Laboratorio di igiene fisiologica Stadium Gate 27 dell'ateneo di Minneapolis per continuarne l'opera. Nel mese di aprile 2015, il giorno del suo novantesimo compleanno, il professore ha concesso a me e a Rossella Galletti un'intervista nel suo studio presso l'Università del Minnesota. Con un certo stupore Galletti ed io ci siamo rese conto che il professore, per sua stessa ammissione, non era ancora a conoscenza del fatto che la dieta mediterranea fosse diventata patrimonio dell'umanità cinque anni prima. Una disinformazione apparente, che potrebbe essere interpretata come effetto di una dicotomia tra l'idea di dieta mediterranea ormai in uso 
nelle scienze biomedicali, che sono andate riducendo progressivamente tale nozione agli aspetti meramente nutrizionali della cosiddetta piramide alimentare. E dall'altra parte quel patrimonio culturale immateriale che l'Agenzia delle Nazioni Unite ha inteso valorizzare, che fa riferimento alle pratiche, alle tradizioni, alle tecniche e alle retoriche che ruotano intorno alla convivialità mediterranea. Escludendo, come non pertinente, ogni considerazione di ordine medico.

Lei è nato in Florida da un padre che era il pastore metodista di Miami Beach. Non volendo seguire le sue orme si è laureato in medicina e poi ha conseguito la specializzazione in cardiologia all'università del Minnesota, dove è entrato nel gruppo di ricerca di Ancel Keys a ventidue anni. Quando è avvenuto il suo primo incontro con la dieta mediterranea?

Credo che il primo episodio veramente significativo sul piano personale sia stato in Dalmazia alla fine degli anni Cinquanta. Ero stato invitato a cena da una famiglia di pescatori. Non se la passavano bene, perché il mare non era affatto generoso. Ciò nonostante avevano imbandito una tavola meravigliosa. Dopo che avevo piluccato qualcosa, sapendo di avere difficoltà nella digestione, rifiutai di mangiare dell'altro. Ma sotto il tavolo mi arrivò un calcio micidiale da Anna Brodaric, la moglie del direttore dell'Istituto di Nutrizione della Jugoslavia, una celebre partigiana con un temperamento fiero e imperioso. Allora chiesi di ridarmi i piatti che avevo rifiutato e così mangiai gamberi, polipo, pesce stella e la notte in qualche modo superai $\mathrm{i}$ postumi dell'abbuffata. Allora imparai la prima regola dell'ospitalità mediterranea. La convivialità è sacra.

Da quando lei è andato in pensione, era il 1995, è diventato lo storico del Seven Countries Study, ha costruito due fondamentali siti web (www.sevencountriesstudy.com; www.epi.umn.edu) dove ci sono centinaia di interviste con i protagonisti delle ricerche sulla dieta mediterranea, bibliografie, articoli scientifici e divulgativi. Ci sono filmati straordinari come quello girato nel 1957 durante lo studio pilota del Seven Countries Study, che consisteva in una messa a punto della metodologia della ricerca e che si è svolto a Nicotera e a Creta. Nella cittadina calabrese una popolazione poverissima, da film neorealista, guarda incuriosita i ricercatori. Ancel e Margaret Keys, accompagnati da Paul White, il cardiologo del Presidente degli Stati Uniti Eisenhower, Alfonso Del Vecchio, Flaminio Fidanza e altri scienziati dell'équipe entrano nelle case dei contadini. In una cantina si vedono due uomini pigiare l'uva con i piedi nudi in un enorme tino, mentre cantano a squarciagola 'O sole mio. Quando il professor Vittorio Puddu, in favore di telecamera e con un microfono in mano, li invita a sottoporsi agli esami biomedicali loro si schermiscono e dichiarano di non avere tempo da perdere. Dopo una breve trattativa uno dei due accetta l'invito. Solleva la sua canottiera e mostra un torace scheletrico, sul quale il dottor White posa il suo stetoscopio. Poco dopo il calabrese viene sottoposto ad un elettrocardiogramma, di seguito agli esami del sangue e in fine racconta la sua storia personale, medica e non solo.

Si tratta di un'etnofiction fortemente condizionata dagli stilemi televisivi del tempo, ai quali si mescola una certa curiositas antropologica à la Alan Lomax, con un vago sapore di propaganda. II che trova qualche giustificazione nel fatto che il filmato serviva per raccogliere fondi e costruire consenso attorno alla vostra spedizione. Ma lo studio delle sette nazioni poi, alla prova dei fatti, è stato davvero un lavoro etnografico da antropologi?

La nostra era un'etnografia a tutti gli effetti, l'unica differenza è che studiavamo popolazioni più ampie e usavamo strumenti che ci fornivano molti dati misurabili. $\mathrm{E}$ quello che abbiamo trovato non è solo un regime nutrizionale che previene le malattie, ma uno stile di vita che promuove un livello altissimo di salute, senza negare alle persone il diritto al piacere e alla felicità. Anche per questo Ancel Keys preferiva usare l'espressione "Stile di vita mediterraneo", perché rendeva meglio l'idea che questo modello sociale è figlio dell'antica civiltà del Mediterraneo. Nasce 
insomma da una cultura stratificata, a differenza della parola dieta che spesso viene intesa in modo restrittivo. Invece le forme di vita hanno a che fare con l'intera dimensione umana, compreso il rapporto con gli altri.

Quindi non avete studiato solo gli usi alimentari, ma anche gli uomini nel loro ambiente naturale e culturale. Non a caso due storici della medicina come Margaret Lock e Vinh-Kim Nguyen nel libro An Anthropology of Biomedicine (2010: 180-182) considerano la vostra ricerca un caso esemplare di quegli esperimenti che vengono definiti "naturali", poiché investigano la vita reale di gruppi di popolazione dislocati in luoghi geograficamente distanti, con abitudini differenti e per periodi di tempo piuttosto lunghi. Condizioni che consentono un'analisi comparativa estesa e preziosa per i risultati che può dare in termini di conoscenza di fenomeni complessi come gli stili di vita.

Noi abbiamo cercato un metodo per studiare scientificamente quello che i popoli del mediterraneo avevano elaborato nel tempo. Cioè uno dei più straordinari sistemi di vita in grado di favorire la salute e la longevità. E con le nostre comparazioni lo abbiamo dimostrato. Poi altri scienziati come Walter C. Willet della Scuola di Salute Pubblica dell'Università di Harvard e Jeremiah Stamler dell'Università North Western di Chicago, hanno dato grande visibilità a questi risultati.

II vostro gruppo di ricerca si è sempre dedicato alla salute pubblica, ma come la definirebbe?

Spesso noi medici ci occupiamo della salute del singolo individuo e ci dimentichiamo dell'influenza esercitata dal livello di educazione e dalla cultura dell'alimentazione che quella persona ha ricevuto dalla sua famiglia e più in generale dalla società. Occuparsi di salute pubblica significa allargare il proprio orizzonte di azione. In un certo senso significa pensare in grande.

\section{L'epidemiologo della nutrizione}

Il dottor Alessandro Menotti è stato prima ricercatore sul campo in tutti i paesi del Seven Countries Study, poi ha ricoperto il ruolo di Project Officer del progetto e membro del Central Staff che aveva sede nel Laboratorio Stadium Gate 27 dell'Università del Minnesota. In seguito ha ricevuto il compito di responsabile della codifica di tutti i dati clinici e della mortalità (follow up), in particolare è stato responsabile delle aree italiane. Inoltre fino al 2006 Menotti è stato Membro del Coordinating Commiter e poi molto attivo con analisi sviluppate in Italia, in Olanda e all'Università del Minnesota, nonché professore di Epidemiologia all'università del Minnesota e a lungo direttore del Laboratorio di Epidemiologia e Biostatistica dell'Istituto Superiore di Sanità. L'ho incontrato per questa intervista a Roma nel giugno del 2015. La sua gentilezza era pari alla sua diffidenza. Troppe volte, mi ha confessato, dai media ha ricevuto grandi delusioni. Giornalisti impreparati e registi indifferenti alla sua natura di ricercatore puro, lontano dal mondo della comunicazione, lo avevano sottoposto a stressantissime sedute di registrazione televisiva. Anche per questo si presenta guardingo e rispondendo alle prime domande, sembra recitare un copione mandato a memoria.

Per cinquant'anni lei ha affiancato Ancel Keys per le analisi statistiche, quali erano le vostre ipotesi di partenza?

Prima ipotesi: stabilire se tra popolazioni diverse, appartenenti a paesi diversi o culture diverse, esistessero reali differenze di prevalenze, incidenze e mortalità per le cardiopatie coronariche e per altre malattie cardiovascolari. Seconda: se queste 
differenze fossero state confermate, allora bisognava stabilire se gli stili di vita, in particolare le differenze di abitudini alimentari, potessero spiegare almeno una parte delle differenze. Terza: se all'interno di singole popolazioni si potessero identificare delle caratteristiche personali, che potessero favorire la predizioni degli eventi. In parole povere, se si potesse capire chi rischiava l'infarto.

Mentre su questa terza ipotesi stavano lavorando anche altri gruppi di ricerca, sulle prime due lavorava solo il nostro gruppo del Seven Countries Study.

Quindi il vostro è stato un lavoro pionieristico.

Eravamo dei pionieri, senza dubbio. Affrontavamo enormi difficoltà operative sul campo, da ogni punto di vista, perché era tutto da inventare. Anche se il professor Keys aveva organizzato, prima dell'inizio dello studio, una lunga serie di studi di tipo metodologico - compreso uno studio pilota in Calabria a Nicotera, nella provincia di Reggio Calabria - per standardizzare tutti i criteri, tutte le misure, tutte le procedure da eseguire. Tutto ciò che non poteva essere standardizzato, non fu adottato. Furono lasciate da parte alcune misure di grande interesse, come ad esempio la glicemia, la trigliceridemia, le radiografie, perché non c'era la possibilità a quei tempi di standardizzare l'esecuzione e la lettura di questi dati.

Che cosa differenzia la patologia geografica della prima metà del Novecento da uno studio epidemiologico longitudinale come il Seven Countries Study?

Il termine patologia geografica è stato coniato per descrivere delle osservazioni sporadiche, fatte da personaggi, di solito strani. Viaggiatori, pionieri, europei trasferiti in aree lontane che senza nessun proposito particolare, hanno raccolto dei dati, hanno fatto delle osservazioni e hanno fatto delle ipotesi intorno a queste osservazioni. E queste ipotesi rappresentano la base di quello che sono stati gli studi epidemiologici longitudinali, che invece hanno un'ipotesi, una tipologia, una struttura e una complessità, come una qualsiasi procedura scientifica. Da questo punto di vista vale sempre la pena ricordare questi precursori.

Il primo è stato Cornelis de Langen, un medico olandese mandato a insegnare medicina interna in Indonesia, che all'inizio del Novecento era una colonia olandese. Lui si divertiva a misurare il colesterolo a tutti. Si accorse che negli indigeni indonesiani, era molto basso rispetto agli olandesi. E si accorse pure che la diagnosi di "Angina Pectoris" - la metto tra virgolette per dire che era un modo per parlare di malattie di cuore, poiché il termine infarto del miocardio non esisteva ancora - era relativamente comune tra gli Olandesi, ma non esisteva tra gli Indonesiani. Allora buttò lì l'ipotesi che la dieta di questi indigeni fosse responsabile del basso livello di colesterolo e che un indice basso di colesterolo fosse il responsabile della mancanza delle malattie cardiache. Ma era un'ipotesi dettata solamente da questa osservazione alimentare e non documentata. Trent'anni dopo un allievo di de Langen, Isidore Snapper, andò a insegnare medicina interna all'Università di Pechino in Cina e fece osservazioni assolutamente simili, col vantaggio di disporre dell'elettrocardiografo. Lui ha documentato che le lesioni da infarto miocardico erano inesistenti tra i Cinesi. Contemporaneamente aveva osservato che questi assumevano una dieta vegetariana, diversissima rispetto a quella dei suoi connazionali olandesi.

Anche io, prima di incontrare Keys, avevo fatto un'osservazione del genere in Libia, dove ho lavorato nell'ospedale governativo di Tripoli dal 1960 al 1962. Avevo notato che i casi d'infarto erano molto più frequenti tra gli Italiani e gli Ebrei, piuttosto che fra gli Arabi Libici. In quegli anni mi imbattei in uno studio della FAO del 1958 
condotto da Giovanni Ferro-Luzzi che descriveva la dieta dei Libici. E mi accorsi che era una dieta strana, povera di calorie, 1.800 al giorno, di cui solo il 13\% era data dai grassi, per lo più vegetali. E anche le proteine erano pochissime. Quando mostrai i miei dati al mio maestro, il professor Vittorio Puddu, allora Primario di Cardiologia e Direttore del Centro per le Malattie Cardiovascolari dell'Ospedale S. Camillo di Roma, mi indirizzò dal professore Ancel Keys, con il quale stava collaborando da qualche anno e venni arruolato subito nello Studio delle Sette Nazioni.

Quindi esistono regimi alimentari salutari quanto quello mediterraneo anche in altre culture?

Certo, anche il Giappone nel nostro studio risultava più mediterraneo della dieta mediterranea. E il Sudafrica pure. Ma in quegli anni chi avrebbe voluto mangiare cinese, giapponese o come le tribù bantu? Keys se lo era posto il problema. Ma era del tutto evidente che un modello nutrizionale del Sud dell'Europa era più proponibile di altri.

Per questo avete inventato l'indice di mediterraneità (MAI)?

Quando il professor Flaminio Fidanza - dal 1965 Professore di Nutrizione Umana all'Università di Perugia, scomparso nel 2012, e tra i primi collaboratori italiani di Ancel Keys - che era il nutrizionista principale del gruppo italiano, creò il cosiddetto indice MAI (Mediterranean Adequacy Index) fece un'operazione d'identificazione di pattern a priori. In altre parole, io stabilisco qual è la dieta buona, poi andiamo a vedere se fa bene oppure no. Lui decise che doveva essere quella di Nicotera del ' 57 , perché pur non avendo Nicotera un follow-up di mortalità completo, aveva caratteristiche molto simili, sotto ogni punto di vista, alle aree della Dalmazia, della Grecia e dell'Italia centrale che avevano invece dei follow-up eccellenti, con bassa mortalità coronariche e bassa incidenza coronarica. Quindi Nicotera venne presa come punto di riferimento e attorno alla dieta di Nicotera fu creato questo indice MAI. Quanto più questo indice è elevato, tanto più un regime alimentare è mediterraneo, quanto più è basso, tanto meno è mediterraneo. Il punto di riferimento, come ho detto, era la dieta del Sud degli anni Cinquanta, che era ricca di pane, pasta, patate, cereali, olio d'oliva, con molta verdura, molta frutta, poca carne, un po' di pesce, pochi derivati del latte. Accompagnata da una quantità modesta di vino e zero dolci.

Durante i lunghi soggiorni di ricerca la vostra équipe ha sperimentato tutti i modi di alimentarsi delle nazioni coinvolte. E nei vostri diari emerge che i due regimi più antitetici erano quello finlandese e quello greco. In cosa differivano?

Quando lavorammo in Grecia c'era l'abitudine di pranzare nelle case dei contadini, che ci preparavano il pranzo per cifre molto modeste. Ci offrivano gli stessi piatti che cucinavano per loro. Tanto pane, tante verdure, soprattutto patate, melanzane, peperoni, zucchine. Pochissima carne, a volte un po' di pollo, un'enormità di frutta e un po' di vino. Questa era la dieta. Naturalmente tutto condito con tanto olio d'oliva. E spesso le verdure erano fritte nell'olio di oliva. Alla fine di questa esperienza ci sentivamo, per così dire, "oliati". Ancel Keys raccontava sempre con stupore e divertimento che a Creta aveva visto i contadini bere un bicchiere di olio extra vergine di oliva per colazione.

Anche in Finlandia, nella Carelia del Nord, andavamo a casa dei contadini che ci preparavano il pranzo, ma l'impressione era completamente diversa. Perché in mezzo alla tavola c'era sempre un tagliere di legno, che assomigliavano a quelli che nel Nord 
d'Italia si usavano per versare la polenta, ma al suo posto c'era una montagna di burro. E appena ci sedevamo a tavola si cominciava a spalmare il burro su enormi fette di pane e si continuava così per tutto il pranzo. Che di solito era composto di un piatto di carne, spesso maiale. Il tutto accompagnato da bicchieroni di latte intero. Allora i Finlandesi, adulti e bambini, bevevano quasi un litro di latte al giorno. E noi alla fine di ogni giornata avevamo la sensazione di esserci "imburrati".

Insomma la diversità culturale, a tavola e non solo, era il vostro terreno di ricerca.

Tutti noi avevamo un'autentica passione per le altre culture. Durante i nostri simposi parlavamo spesso di cucina, di arte, di letterature. Ancel Keys aveva una notevole cultura di tipo storico-geografico e archeologico, d'altra parte aveva anche lui viaggiato molto e aveva avuto tanti incontri con culture e ambienti diversi.

Durante la mia ricerca per scrivere il libro La dieta mediterranea. Mito e storia di uno stile di vita, mi sono chiesta chi avesse inventato la definizione Dieta Mediterranea. Quando ho letto questa espressione a pagina 11 del libro di Ancel e Margaret Keys How to eat well and stay well: The Mediterranean Way, pubblicato nel 1975, ho ipotizzato che quella fosse la prima volta che in un testo a stampa compare questa felice espressione. Ora chiedo a lei, quand'è che avete inventato questo nome?

Questa è una storia difficile da raccontare, perché anche noi siamo molto incerti. Effettivamente, il sottotitolo di quel libro indica per la prima volta "The Mediterranean Way", lo stile mediterraneo. Ma si trattava di un libro per il largo pubblico, non di una pubblicazione scientifica. Nove anni dopo, nel 1984, la professoressa Anna Ferro-Luzzi dell'Istituto Nazionale di Nutrizione di Roma condusse un esperimento sulla dieta mediterranea nel Cilento, nella località dove $\mathrm{i}$ coniugi Keys avevano scelto di vivere dopo la pensione. La supervisione della ricerca fu proprio di Ancel Keys. Non a caso tra i 48 soggetti analizzati molti erano suoi conoscenti e tra questi la sua cuoca Delia Morinelli. Lo studio consistette nel sostituire la dieta abituale a base di olio d'oliva con la western diet, che invece è ricca di grassi animali. Nello stesso periodo un campione simile in Finlandia fece l'inverso, sostituendo la dieta tradizionale con quella cilentana. L'esito fu un innalzamento evidente della colesterolemia tra i Cilentani e un abbassamento tra i Finlandesi. I risultati vennero pubblicati nell'American Journal of Medical Nutrition in un articolo intitolato Changing the Mediterranean diet: effects on blood lipids. Quello è il primo articolo scientifico internazionale a usare il termine dieta mediterranea.

Va sottolineato però che in quell'articolo gli autori, tra cui il grande clinico Mario Mancini e Pasquale Strazzullo, per far capire meglio a quale modello alimentare si riferissero, usano anche l'espressione "dieta di tipo mediterraneo". A riprova del fatto che il nome dieta mediterranea era ancora poco noto e andava spiegato al lettore.

Allora possiamo dire che, come spesso accade alle grandi idee che sono collettive e non hanno un momento iniziale, ma piuttosto un momento sorgivo, nel caso della dieta mediterranea è iniziato tutto con il vostro Seven Countries Study. Cui poi sono seguiti studi internazionali come MONICA, SCORE, EPIC ecc.

Questo è un bel modo di interpretare, perché qui stiamo ormai parlando di una storia che risale a mezzo secolo fa e nel frattempo tante cose si sono mosse, si son sviluppate, si sono modificate, fino al consolidarsi di questa idea. Che ora è entrata nel lessico comune e perfino in quello di un'istituzione prestigiosa come l'UNESCO. Che ha il merito di far capire che cosa è la cultura e diffonde le grandi idee.

Dove sono finiti tutti i materiali prodotti dalla vostra ricerca, come i diari alimentari dei ferrovieri di Roma e di Minneapolis, i dati delle coorti italiane di Monte Giorgio e Crevalcore, 
quelle greche di Atene, Heraklion, Corfù, quella olandese di Zutphen, quelle in Dalmazia e Slovenia, quella finlandese della Carelia del Nord e quelle statunitensi di Minneapolis e Saint Paul, nonché dell'isola nipponica di Kyushu?

L'Università del Minnesota affittò un hangar per conservare tutti i documenti che avevamo raccolto. Quando finirono i finanziamenti ne buttarono una parte, io portai tutto quel che potei a Roma all'Istituto Superiore di Sanità, ma prima di andare in pensione mandai tutto in Olanda da un collega. Ad un certo punto anche lui non riuscì più a trovare $\mathrm{i}$ fondi per questo enorme archivio. E tutta la nostra documentazione cartacea riferita a 12 mila persone, raccolta certosinamente per quasi cinquant'anni, è andata distrutta. Cancellata per sempre. Abbiamo le sintesi dei risultati su supporti informatici, ma non abbiamo più né i dati grezzi, che un domani avrebbero potuto essere interrogati in modi nuovi, né i diari alimentari, né le anamnesi mediche del popolo del Seven Countries Study. Un vero delitto! Solo la Finlandia ha conservato tutto e ha un programma di salvaguardia finanziato per $\mathrm{i}$ prossimi cinquant'anni.

Concludiamo la nostra conversazione condividendo il rammarico del fatto che mentre noi Italiani abbiamo insegnato ai Finlandesi uno stile di vita più sano e sostenibile, sia da un punto di vista ambientale che sociale, di contro il governo scandinavo ha molto da insegnare all'Italia nell'ambito della conservazione del patrimonio culturale.

\section{Conclusioni}

Il patrimonio culturale immateriale della dieta mediterranea che emerge dalle interviste che ho condotto presso alcuni scienziati "superstiti" del Seven Countries Study, come Henry Blackburn e Alessandro Menotti, nonché presso due testimoni particolari come Carrie Keys D'Andrea e Anna Ferro-Luzzi, mostra che all'origine della individuazione di questo pattern culturale vi era una spiccata propensione verso una sorta di antropologia spontanea. L'ipotesi principale di questo studio medicoetnografico, infatti, si fondava sull'idea che la vita quotidiana e i costumi alimentari dei popoli del Mediterraneo custodissero la ricetta per una vita sana e longeva. E i successivi sviluppi del SCS hanno confermato l'ipotesi di partenza ricorrendo agli strumenti specifici della ricerca bio-medica, ma coniugandoli opportunamente con un approccio etnografico, ancorché amatoriale (Kromhout et al. 1993).

Inoltre Ancel e Margaret Keys, con l'équipe internazionale del Seven Countries Study, ritennero che il modello culturale della dieta mediterranea potesse essere esportato e promosso nel mondo anglo-americano per promuovere una politica di salute pubblica, alla portata di tutti i ceti, con lo scopo principale di prevenire le malattie cardiovascolari. Proprio per consentire un'identificazione immediata di questo stile di vita, come emerge chiaramente da queste testimonianze, nel 1975 i coniugi Keys coniarono l'espressione dieta mediterranea nel loro best seller How to Eat Well and Stay Well. The Mediterranean Way, di fatto inaugurandone l'uso. La prima volta invece che questa espressione venne utilizzata in ambito scientifico è in un articolo apparso nel 1984 sull'American Journal of Clinical Nutrition dal titolo Changing the Mediterranean diet: Effects on blood lipids, che - come ho già detto - riferiva di uno studio compiuto in Cilento da Anna Ferro-Luzzi e altri ricercatori, sotto la supervisione di Ancel Keys. Sostituendo la dieta tradizionale dei Cilentani con la western diet risultò presto evidente che il tasso di colesterolo nel sangue dei soggetti esaminati saliva inesorabilmente, e 
che pertanto non vi era alcuna predisposizione genetica in quella popolazione che giustificasse la ridotta colesterolemia e conseguentemente la scarsa incidenza delle malattie cardiovascolari. Rendendo così evidente il fatto che a mantenere in buona condizione di salute la popolazione cilentana fosse essenzialmente lo stile di vita. Va inoltre sottolineato che lo studio di Ferro-Luzzi sul Cilento ha contribuito, in maniera indiretta, alla identificazione nel senso comune di quella area della Campania con la "scoperta" della dieta mediterranea. Ed è proprio questa una delle ragioni principali per cui l'UNESCO ha riconosciuto quel territorio e in particolare il comune di Pollica, dove Ancel e Margaret Keys vissero per trentacinque anni, quale "comunità emblematica" della dieta mediterranea (Scovazzi, Ubertazzi, Zagato 2012; Mariotti 2013). Altrimenti molte altre località del Belpaese, con stili di vita e pratiche alimentari simili a quelle cilentane, avrebbero potuto, legittimamente, rivendicare un ruolo analogo.

Questa ricostruzione genealogica del termine dieta mediterranea va letta come un esercizio di memoria e inteso come un contributo alla salvaguardia di una grande storia a partire da un dettaglio tutt'altro che marginale qual è il nome. Si tratta di un lavoro di ricerca svolto nella convinzione che avesse ragione Samuel Johnson quando scriveva che «la vera arte della memoria è l'arte dell'attenzione».

\section{BIBLIOGRAFIA}

Blackburn H., 2017 «Invited Commentary: 30-Year Perspective on the Seven Countries Study», American Journal of Epidemiology, Vol. CLXXXV, No. XI doi: 10.1093/aje/kwX071

Bloch M., 1969 Apologia della storia o mestiere di storico, Einaudi, Torino.

Boccardi V., Calvani R., Limongi F. et al., 2018 «Consensus paper on the "executive summary of the international conference on mediterranean diet and health: a lifelong approach", an italian initiative supported by the mediterranean diet foundation and the menarini foundation», Nutrition, https://doi.org/10.1016/j.nut.2017.12.002.

Dixon J.L., 2015 Genius and partnership. Ancel and Margaret Keys and the Discovery of the Mediterranean Diet, Joseph L. Dixon Publishing, New Brunswik, NJ.

Ferro-Luzzi A., Strazzullo P., Scaccini C. et al., 1984 «Changing the Mediterranean diet: Effects on blood lipids», in American Journal of Clinical Nutrition, XL(V):1027-37.

Geertz C., 1973 The Interpretation of Cultures, Basic Books, New York; trad. it. 1987, Interpretazione di culture, il Mulino, Bologna.

Grimaldi P., 2012 Cibo e rito: il gesto e la parola nell'alimentazione tradizionale, Sellerio, Palermo.

Grimaldi P., Porporato D., 2012 Granai della memoria: manuale di umanità 2.0, Università di Scienze Gastronomiche, Pollenzo. 
Herder J.G., 1774 Auch eine Philosophie der Geschichte zur Bildung der Menshheit, in Id., 1891, Sämtliche Werke, a cura di B. Suphan, vol. V, Weidmannsche Buchhandlung, Berlin: 475-586; trad. it. 1951, Ancora una filosofia della storia per l'educazione dell'umanità, Einaudi, Torino.

Keys A., Keys M., 1959 Eat well and stay well, Doubleday, Garden City, N.Y.; trad. it. 1962, Mangiar bene e stare bene (con la dieta mediterranea), Piccin, Padova.

Keys A., Keys M., 1960 Eat well and stay well: Special British edition revised and adapted by dr Magnus Pyke, Hodder \& Stoughton, London.

Keys A., Keys M., 1967 The benevolent bean, Doubleday, Garden City, N.Y.

Keys A., Keys M., 1975 How to eat well and stay well: The Mediterranean way, Doubleday, Garden City, N.Y.

Keys A., Keys M., 2017 La dieta mediterranea. Mangiare bene e stare bene, Slow Food - Giunti, Firenze.

Keys A. et al., 2017 «The diet and 15-year death rate in the Seven Countries Study», in American Journal of Epidemiology 1986, CXXIV: 903915. REPRINTED as Hictoric Article. in American Journal of Epidemiology 2017, CLXXXV: 1130-1142.

Kromhout D. et al. (eds), 1993 The Seven Countries Study. A scientific adventure in cardiovascular disease epidemiology, Brouwer Offset bv, Utrecht.

Kromhout D. et al., 1995 «Dietary saturated and trans fatty acids and cholesterol and 25-year mortality from coronary heart disease: the Seven Countries Study», Preventive Medicine, XXIV: 308-315.

Lock M., Nguyen V.-K., 2010 An anthropology of biomedicine, Wiley-Blackwell, Malden (USA), Oxford (UK), Chichester-West Sussex (UK).

Mariotti L., 2013 «La convenzione sul patrimonio intangibile e i suoi criteri tra valorizzazione, tutela e protezione», in «Voci. Annuale di scienze umane», X: 88-97.

Menotti A., Kromhout D. et al., 2017 «Baseline fatty acids, food groups, a diet score and 50-year all-cause mortality rates. An ecological analysis of the Seven Countries Study», Annals of Medicine, XLIX: 718-727.

Moro E., 2013 L'invenzione della dieta mediterranea. Genealogia medico-cultu- rale di un modello alimentare, in «am - rivista della società italiana di antropologia medica», 35-36, ottobre: 13-39.

Moro E., 2014 La dieta mediterranea. Mito e storia di uno stile di vita, Il Mulino, Bologna.

Moro E., 2016 «The Mediterranean Diet from Ancel Keys to the UNESCO Cultural Heritage. A Pattern of Sustainable Development between Myth and Reality», Procedia - Social and Behavioral Sciences, CCXXIII: 655-661.

Moro E., Niola M., 2017 Andare per i luoghi della dieta mediterranea, Il Mulino, Bologna.

Petrillo P.L. (ed.), 2018 The Legal Protection of Intangible Cultural Heritage. A Comparative Perspective, Springer, Berlino.

Pett K.D. et al., 2017a The Seven Countries Study. Reorienting to the primary sources serves to deconstruct longstanding myths surrounding the seminal Seven Countries Study, doi: 10.1093/eurheartj/ehx603, Cardio Pulse, Eur Heart J.: 3119-3121.

Pett K.D. et al., 2017b Ancel Keys and the Seven Countries Study: An Evidence-based Response to Revisionist Histories. WHITE PAPER Commissioned by The True Health Initiative With emphasis on 
primary source material, historical records, and review/critique by Seven Countries Study investigators. August 1, http://www.truehealthinitiative.org/.

Remotti F., 1990 Noi primitive. Lo specchio dell'antropologia, Bollati Boringhieri, Torino.

Scovazzi T, Ubertazzi B., Zagato L. (a cura di), 2012 Il patrimonio culturale intangibile nelle sue diverse dimensioni, Giuffrè, Milano.

Solfrizzi V. et al., 1999 «Monounsaturated fattyacids intake protects against age-related cognitive decline», Neurology, LII: 1563-1569.

Teti V., 1999 Il colore del cibo. Geografia, mito e realtà dell'alimentazione mediterranea, Meltemi, Roma.

Toshima H., Koga H., Blackburn H. (eds), 1994 Lessons fo Science from the Seven Countries Study. A 35Year Collaborative Experience in Cardiovascular Disease Epidemiology, Springer, Berlino.

Truswell A.S., 2010 Cholesterol and beyond: The research on the diet and coronary heart disease 1900-2000, Springer, Dondrecht.

\section{RIASSUNTI}

L'autrice riscostruisce la genealogia del termine "dieta mediterranea" e la sua introduzione nel lessico scientifico e nel senso comune occidentali. Questa espressione, infatti, risale solo alla seconda metà del Novecento. Attraverso una serie di testimonianze dirette e un'analisi delle fonti, Moro mette in evidenza il fatto che ad inventare questa fortunata espressione sono stati Ancel Keys e Margaret Haney Keys, scopritori di questo stile di vita, che attraverso il Seven Countries Study (1958) ne hanno dimostrato per primi i benefici sulla salute. Con il libro How to Eat Well and Stay Well. The Mediterranean Way (USA 1975) i Keys introducono nel lessico occidentale il nome dieta mediterranea. Tale termine entra invece ufficialmente nel lessico scientifico grazie ad una ricerca sul rapporto tra dieta e colesterolo, fortemente voluta da Ancel Keys, condotta da Anna Ferro-Luzzi et all. in Cilento-Italia (oggi comunità UNESCO della dieta mediterranea) e pubblicata nel 1984 sul prestigioso American Journal of Clinical Nutrition AJCN.

The author reconstructs the genealogy of the term "Mediterranean diet" and its introduction into the scientific lexicon and in the western general sense. Indeed, this expression dates back only to the second half of the twentieth century. After collecting witnesses and analyzing the sources, Moro points out that Ancel Keys and Margaret Haney Keys, the discoverers of this way of life, invented this successful expression. They showed the health benefits of the Mediterranean Diet through the Seven Countries Study (1958). Thanks to the book How to Eat Well and Stay Well. The Mediterranean Way (USA 1975) the Keys introduced the expression "Mediterranean diet" into the western lexicon. However, this term officially enters into the scientific lexicon thanks to research carried out on the relationship between diet and cholesterol. The study was strongly desired by Ancel Keys and performed by Anna Ferro-Luzzi et all. in Cilento-Italy (today one of the UNESCO communities of the Mediterranean diet). This research was published in 1984 in the prestigious American Journal of Clinical Nutrition AJCN.

\section{INDICE}

Parole chiave : dieta mediterranea, Seven Countries Study, Keys (Ancel), Ferro-Luzzi (Anna), Cilento (Italia)

Keywords : Mediterranean diet, Seven Countries Study, Keys (Ancel), Ferro-Luzzi (Anna), Cilento (Italy) 


\section{AUTORE}

\section{ELISABETTA MORO}

Università di Napoli Suor Orsola Benincasa elisbetta.moro[at]unisob.na.it 